\title{
Sepsis in Post Cardiac Surgery Patient - A Rare Cause
}

\author{
Sarvesh Pal Singh ${ }^{1} \quad$ Sukhjeet Singh ${ }^{1}$ \\ 1Department of Cardio-Thoracic and Vascular Surgery, \\ Cardio-Thoracic Sciences Center, All India Institute of \\ Medical Sciences, New Delhi, India
}

\begin{abstract}
Address for correspondence Sarvesh Pal Singh, DM, Department of Cardio-Thoracic and Vascular Surgery, C.N. Center, All India Institute of Medical Sciences, Room No. 2, 8th Floor, New Delhi 110029, India (e-mail: sarveshpal.singh@gmail.com).
\end{abstract}

J Card Crit Care TSS 2018;2:39-40
Abstract
Keywords
- arthritis
- cardiac
- septic
- surgery

A 32-year-old patient who underwent cardiac surgery and developed sepsis in the postoperative period is described. Septic arthritis of knee joint as a rare cause of sepsis in the post cardiac surgery patient is discussed. The role of routine examination of joints in patients under evaluation for sepsis is emphasized.

\section{Introduction}

The yearly incidence of septic arthritis is about 2 to 10 per 100,000 in general population. Staphylococcus aureus is the most commonly isolated causative organism in the adults. Other causative organisms include Group A beta hemolytic streptococci, group B, C, and G (in immunocompromised hosts, gastrointestinal or genitourinary infections), gram negative bacilli (in Injection drug users,elderly and severely immunocompromised hosts), Haemophilus influenzae (newborns), and Gonococcus (in young sexually active individuals). Antibiotics used to treat are guided by the Grams staining of the aspirate obtained and later revised according to the culture and sensitivity results. For gram positive cocci, vancomycin or a beta lactamase resistant antibiotic is administered whereas for gram negative bacilli an aminoglycoside or a third generation cephalosporin is administered. The mortality is 10 to $16 \%$ despite treatment. The incidence of permanent damage to joint is as high as $50 \%$.

\section{Case Report}

A 32-year-old male (weight $42 \mathrm{~kg}$ and height $160 \mathrm{~cm}$ ) diagnosed with rheumatic heart disease (RHD) having severe mitral stenosis, moderate aortic regurgitation, severe tricuspid regurgitation, and severe pulmonary artery hypertension presented to the emergency department in an unresponsive state, with tachypnea and shock. In view of his congestive heart failure, the patient underwent emergency tracheal intubation and initiation of mechanical ventilation with tidal volume of $250 \mathrm{~mL}$, respiratory rate of $22 / \mathrm{min}$; inspiratory: expiratory (I:E) ratio of $1: 2$; and Fraction of inspired oxygen $\left(\mathrm{FiO}_{2}\right)$ of $100 \%$. A central venous catheter was placed in the right internal jugular vein, and infusions of dobutamine, dopamine, and noradrenaline were started in the doses of 5, 5 , and $0.05 \mu \mathrm{g} / \mathrm{kg} / \mathrm{min}$, respectively, to attain a mean arterial pressure of $65 \mathrm{~mm} \mathrm{Hg}$. The neurological status of the patient could not be assessed as he was paralyzed for airway intubation. A preoperative arterial blood gas (ABG) analysis revealed metabolic acidosis (pH 7.24), hyponatremia $(120 \mathrm{mEq} / \mathrm{dL})$, and partial pressure of oxygen $\left(\mathrm{pO}_{2}\right)$ and partial pressure of carbon dioxide $\left(p \mathrm{CO}_{2}\right)$ of 110 and $47 \mathrm{~mm} \mathrm{Hg}$, respectively. His preoperative investigations showed total leukocyte count (TLC) $16,100 / \mu \mathrm{L}$, hemoglobin (HB) $5 \mathrm{~g} \%$, hematocrit (Hct) $15 \%$, and platelet count of $330,000 / \mu \mathrm{L}$. His temperature at the time of presentation was normal. In view of patient's moribund status, he was immediately taken up for surgery after written informed consent from his brother.

The patient underwent a double-valve (mitral and aortic) replacement, tricuspid valve repair, and left atrial (LA) clot removal without any adverse event and was shifted to the intensive care unit (ICU) on intravenous infusions of dopamine, dobutamine, adrenaline, and noradrenaline in doses of 10,5 , 0.2 , and $0.2 \mu \mathrm{g} / \mathrm{kg} / \mathrm{min}$, respectively. In the ICU, there were signs of low cardiac output and poor peripheral perfusion. The lactate level and base deficit were 10 and $-12 \mathrm{mmol} / \mathrm{L}$, respectively. On transthoracic echocardiography, the left ventricular ejection fraction (LVEF) was 15\% with moderate right ventricular (RV) dysfunction and pulmonary arterial systolic pressure (PASP) of $50 \mathrm{~mm} \mathrm{Hg}$. An intra-aortic balloon pump was inserted for hemodynamic stabilization. Neurological status, again, could not be assessed because of anesthesia
DOI https://doi.org/

10.1055/s-0038-1671680.

ISSN 2457-0206.
Copyright @2018 Official Publication of The Simulation Society (TSS), accredited by International Society of Cardiovascular Ultrasound (ISCU).
License terms

()(1) $\ominus \circledast$ 
and residual paralysis from neuromuscular blocking agents. Antibiotic prophylaxis was provided with intravenous ceftazidime $1 \mathrm{~g}$ and ofloxacin $200 \mathrm{mg}$ twice daily.

On postoperative day (POD) 1, the patient developed a highgrade fever $\left(103^{\circ} \mathrm{F}\right)$. A complete work-up of fever with two sets of blood culture, urine culture, chest drain fluid culture, and culture of endotracheal washings was sent. The Glasgow Coma Scale (GCS) of patient was E1M1Vet. On POD 2, the GCS improved to E4M6Vet, and temperature decreased to $100^{\circ} \mathrm{F}$. On POD 3, the temperature was still between $100^{\circ} \mathrm{F}$ and $101^{\circ} \mathrm{F}$, and the GCS deteriorated again to E1M1Vet. In view of clinical deterioration of neurological status, a non-contrast computed tomography (NCCT) head was performed on POD 3, which showed multiple small infarcts in the cortical watershed areas. All samples sent for cultures did not grow any organisms. Repeat cultures were sent from all possible sites. In view of turbulent postoperative course and GCS of E1M1Vet, a percutaneous tracheostomy (PCT) was performed on POD 5. On POD 6, during routine examination, the left knee of the patient was found to be swollen. On examination, it appeared inflamed and tense. A diagnostic tap of the knee joint was performed which revealed frank pus in the knee joint ( - Fig. 1). Intravenous teicoplanin, meropenem, and metronidazole were started for septic arthritis. The culture of pus came positive for Staphylococcus aureus. However, the patient's clinical condition deteriorated very fast, and he expired within 24 hours of the diagnosis of septic arthritis.

\section{Discussion}

Septic arthritis has been described in patients with heart transplant, bacterial endocarditis, and cardiac sarcoidosis. ${ }^{1-3}$ We could not find any report describing the diagnosis of septic arthritis in the postoperative period after cardiac surgery. In our case, we did not suspect infection as the cause of fever in the immediate postoperative period (up to 24 hours). Systemic inflammatory response is a well-established sequelae of cardiopulmonary bypass, and fever in the immediate post cardiac surgery period is quite often ascribed to systemic inflammatory response syndrome (SIRS). ${ }^{4}$ In our patient, however, when the fever persisted for more than 24 hours postoperatively, a proper sepsis workup was sent. The total leukocyte count in the immediate postoperative period had low positive predictive value for sepsis because the surgical inflammation caused an increase in the TLC for initial 24 to 48 hours. A NCCT brain was done to rule out any structural neurological cause for his low GCS score on POD3. The NCCT brain revealed infarcts in cortical watershed areas indicating severe hypoperfusion as the cause for infarcts. In anticipation of prolonged mechanical ventilation (low GCS score and borderline hemodynamic status), we performed an early PCT as our ICU protocol. As the source of sepsis was very rare in our patient, all other cultures turned up negative. In retrospection, it is difficult to contemplate whether

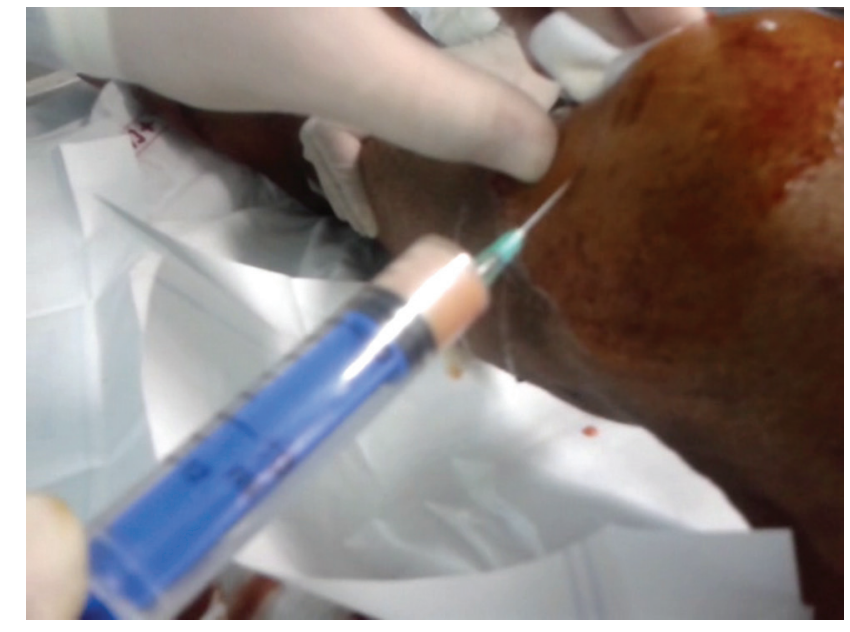

Fig. 1 Pus being aspirated from the knee joint

the infection (of the left knee) was present preoperatively and was the main cause for the symptoms of the patient, or the patient developed perioperative infection at a rare site (left knee) which caused the septic shock. The native valve morphology, histopathology, and culture results in our patient excluded infective endocarditis as the cause of sepsis. The most common bacterium to cause septic arthritis is S. aureus. ${ }^{5}$ In our patient, there was no risk factor (trauma, diabetes mellitus, hypertension, surgery of knee, diseased knee joint, and immunosuppression) present for the development of septic arthritis.

In conclusion, septic arthritis may be present in a patient presenting with congestive heart failure. The examination of joints in a post cardiac surgery patient, who is being evaluated for sepsis, is imperative.

\section{Conflict of Interest}

None.

\section{References}

1 Martin-Santos JM, Alonso-Pulpon L, Pradas G, et al. Septic arthritis by Salmonella enteritidis after heart transplantation. J Heart Transplant 1987;6(3):177-179

2 Martínez Oviedo A, Gracia Sánchez P, Pueo E, Chopo JM. [Septic arthritis as an initial manifestation of bacterial endocarditis caused by Staphylococcus aureus] Med Interna 2006;23(4): 184-186

3 Marcos Vidal JM, Soto Mesa D, Montes Armenteros A, Baticón Escudero PM. [Posterior lumbar plexus block for surgery to treat septic arthritis of the hip in a patient with cardiac sarcoidosis] Rev Esp Anestesiol Reanim 2010;57(4):258-259

4 Laffey JG, Boylan JF, Cheng DC. The systemic inflammatory response to cardiac surgery: implications for the anesthesiologist. Anesthesiology 2002;97(1):215-252

5 Muñoz-Egea MC, Blanco A, Fernández-Roblas R, et al. Clinical and microbiological characteristics of patients with septic arthritis: a hospital-based study. J Orthop 2014;11(2):87-90 